http://jmscr.igmpublication.org/home/ ISSN (e)-2347-176x ISSN (p) 2455-0450

crossref DOI: https://dx.doi.org/10.18535/jmscr/v9i11.37

\author{
(D) Journal Of Medical Science And Clinical Research \\ IGM Publication \\ An official Publication of IGM Publication
}

\title{
Unveiling the Uncanny- Unusual Hemorrhagic Complications of Anticoagulant Therapy - Case Series
}

\author{
Authors \\ Dr Mangalanandan.S ${ }^{1^{*}}$, Dr Amith Itty ${ }^{2}$, Dr Jose ${ }^{3}$, Dr Geena Benjamin 4 \\ ${ }^{1}$ Associate Professor Pushpagiri Medical College Dpartment of Radiodiagnosis \\ ${ }^{2}$ Assistant Professor Pushpagiri Medical College \\ ${ }^{3}$ Senior Resident Pushpagiri Medical College \\ ${ }^{4}$ Professor and HOD Pushpagiri Medical College \\ *Corresponding Author \\ Dr Manalanandan.S
}

\section{Introduction}

The modern clinical practice would be incomplete without the use of multitude of anticoagulants. These anticoagulants are however associated with a definite risk of hemorrhage. The risk of hemorrhage increases with advanced age and the comorbidity status of the patient. The choice of anticoagulants and the duration and dosage are also seen to have an effect on the possibility of developing a hemorrhagic complication. The clinical manifestations are often deceiving and often cross sectional imaging is mandatory for effective diagnosis of such hemorrhagic complications. Prompt identification and diagnosis these complications aid the clinicians to act on time and take necessary steps for timely intervention. In some of the cases interventional procedures may be needed. The choice of the modality may also be influenced by the imaging findings. Few such characteristic cases are presented here before you giving preference to the images.

Large number of patients are being treated using anticoagulants for an array of thromboembolic disorders. Considering the large volume of patients under anticoagulant therapy, the proportion of patients experiencing hemorrhagic complications would be minimal. Although the proportion is minimal, the actual number of cases is quite high. Most of these cases turn out to have lethal outcome. ${ }^{(1-3)}$ Actually any organ system may be involved in hemorrhagic complications of anticoagulants. In many cases, the bleeding site may be obvious from physical examination. But the true existence of the hematoma and possibility of active bleed can only be ascertained with the help of cross sectional imaging. The cross sectional imaging studies would also throw some light into the underlying pathology if contrast enhanced study is performed.

\section{Anticoagulant Treatment Modalities}

Heparin, low molecular heparin and its derivatives are the mainstay of anticoagulant therapy. Heparin binds and activate antithrombin III. This results in the inhibition of thrombin and the intrinsic pathway as a whole. Usually acute thromboembolic events like acute DVT and pulmonary embolism are the ones most benefitted 
by heparin. However the anticoagulant effect of heparin is short lived. It can be monitored by APTT. At times of emergency and toxicity, the effects of heparin can often be easily reversed by Protamine sulphate.

In contrast to this, low molecular weight heparins mainly inhibit factor Xa. It has longer half life and tends to remain in the system for longer time duration.

Warfarin is the most widely used anticoagulant which acts by preventing the formation of vitamin $\mathrm{K}$ dependent clotting factors. This usually leads to depletion of extrinsic pathway. Warfarin is often used for long term indications as in valvular heart disease. ${ }^{(4)}$

\section{Incidence and Risk Factors}

The incidence of anticoagulant related hemorrhage varies from $1 \%$ to $7 \%$ per year round the world. $^{(3)}$ Advanced age, cardiac, cerebrovascular, renal, hepatic diseases and other medications are important risk factors ${ }^{(5)}$. Intravenous heparin therapy further increases the risk to $2 \%-3 \%$ per day out of which $0.05 \%$ to $0.8 \%$ cases may go in for fatal outcome ${ }^{(5,6)}$.

Patients on regular hemodialysis are under even higher risks owing to the poorly functioning platelets and repeated heparin administration at each session of dialysis. ${ }^{(7)}$

Low molecular weight heparin is often associated with lower risks of bleeding rates.

Long term warfarin therapy may also lead to hemorrhagic complications, especially when the INR ratio is not monitored properly. The target INR is accepted as $>3$ globally, however varies for various clinical conditions. The incidence of hemorrhagic complications for these patients is however $0.4 \%$ to $3 \%$ per year ${ }^{(2)}$. The incidence of fatal outcome is $0.6 \%$ while major and minor bleeding complications are $3 \%$ and $9.6 \%$ respectively. It has been observed that the risk of major complications is more (10 times) in the first month of treatment. ${ }^{(3,5,6)}$

\section{CT and MR Acquisition Protocols}

Both CT and MR studies are undertaken for the patients. Multidetector CT images are procured by the GE OPTIMA 128 SLICE machine. All CT studies include a mandatory unenhanced series followed by arterial and venous images. Arterial phase may be acquired using an empiric start delay or with the use of an automatic bolustriggering software with the region of interest placed in the aorta using a 100 Hounsfield units (HU) cutoff. The empiric delay for arterial images is often timed at $25-30$ seconds and the venous images are timed at 45 - 50 seconds. Contrast enhanced images may not be acquired in all the studies and depends on the clinical setting. Contrast images are not done whenever an impaired renal functional status and contrast allergy are reported. In unenhanced images, the characteristic hyperdensity of fresh blood and hematocrit sign are easily identifiable. In contrast images, active contrast extravasation is suggestive of active bleed.

Our in house MRI machine is GE 1.5T SIGNA. The MRI acquisition protocol includes gradient images, T1, T2 and MERGE sequences. Hemorrhagic manifestations can be identified as blooming on MERGE sequences and hyperdensity on $\mathrm{T} 1$ images.

Common sites for developing hematoma in anticoagulant therapy include intracranial, intramuscular and intra abdominal locations. Infrequent sites include perinephric, retroperitoneal, intramural hematoma in bowel and $\operatorname{aorta}^{(8-10)}$. We present some uncommon locations of developing hematomas and its appearances following anticoagulant therapy, appropriating the need for radiologists to be aware of the uncanny sites as well in evaluation of hematoma in post anticoagulant therapy.

\section{Cases}

\section{Perinephric Bleed}

This is a 53 year old lady who presented with left sided abdominal pain and hypotension. She had undergone angiography with stenting few months 


\section{JMSCR Vol||09||Issue||11||Page 187-195||November}

back and is on warfarin now. Since she had atrial fibrillation, she was put on Warfarin. The CECT abdomen revealed a hypodense collection in the left subcapsular and perinephric region which measures approximately $7 \times 5 \times 7 \mathrm{cms}$. CC). The collection is seen to extend acrossthe midline medially, into the perisplenic region superiorly and into the pelvis inferiorly. There isevidence of contrast extravasation in arterial phase of contrast study with gradual increase inextent and pooling of the contrast in venous and delayed phases. However renal arteries showed no evidence of contrast extravasation.
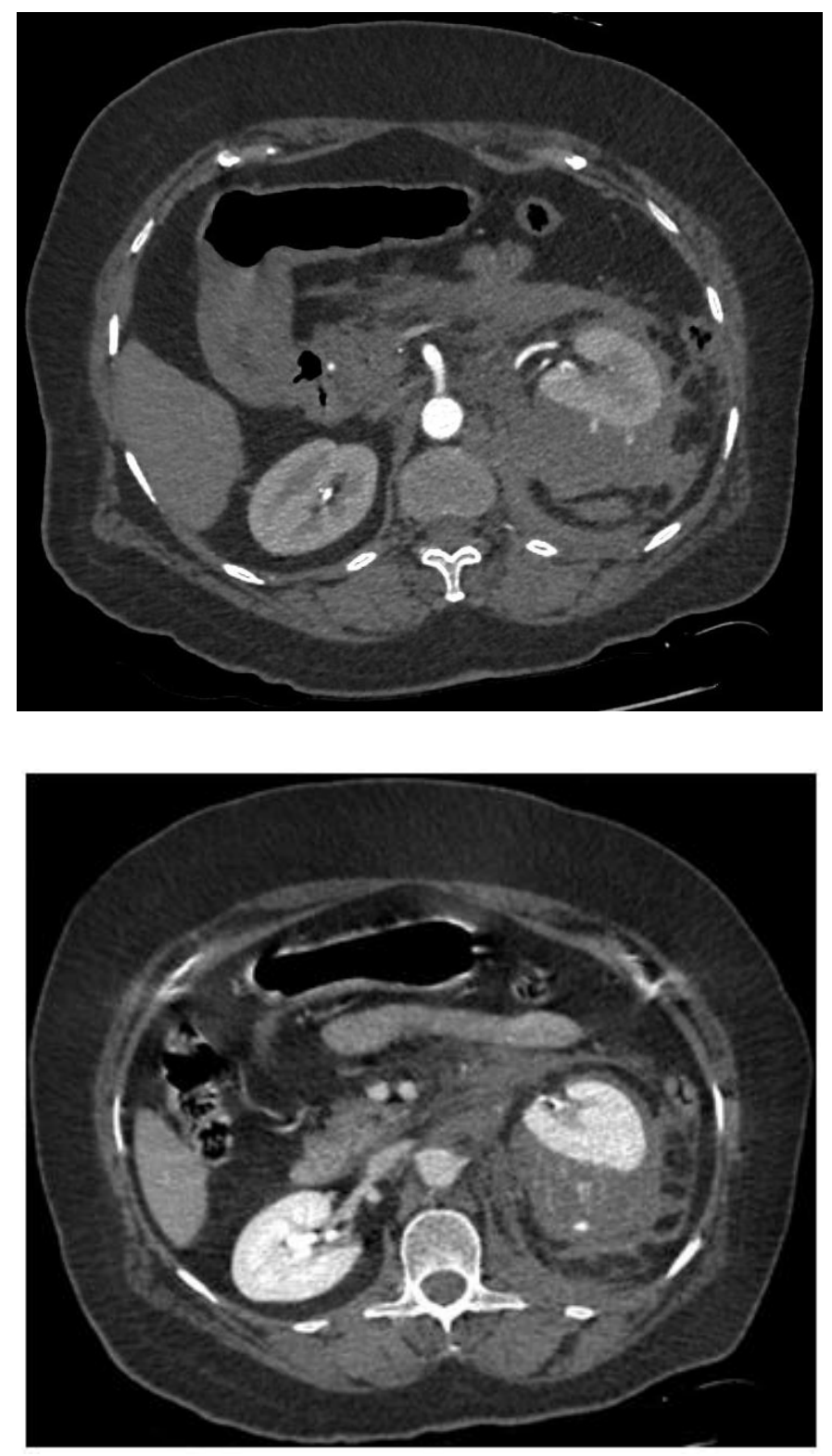
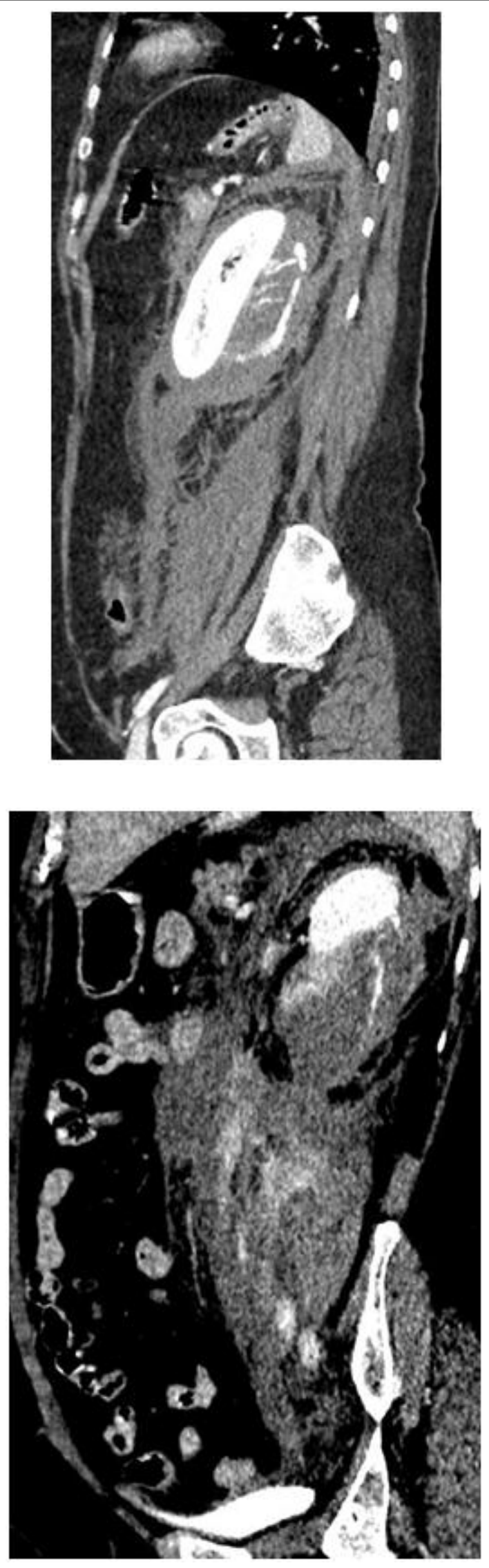


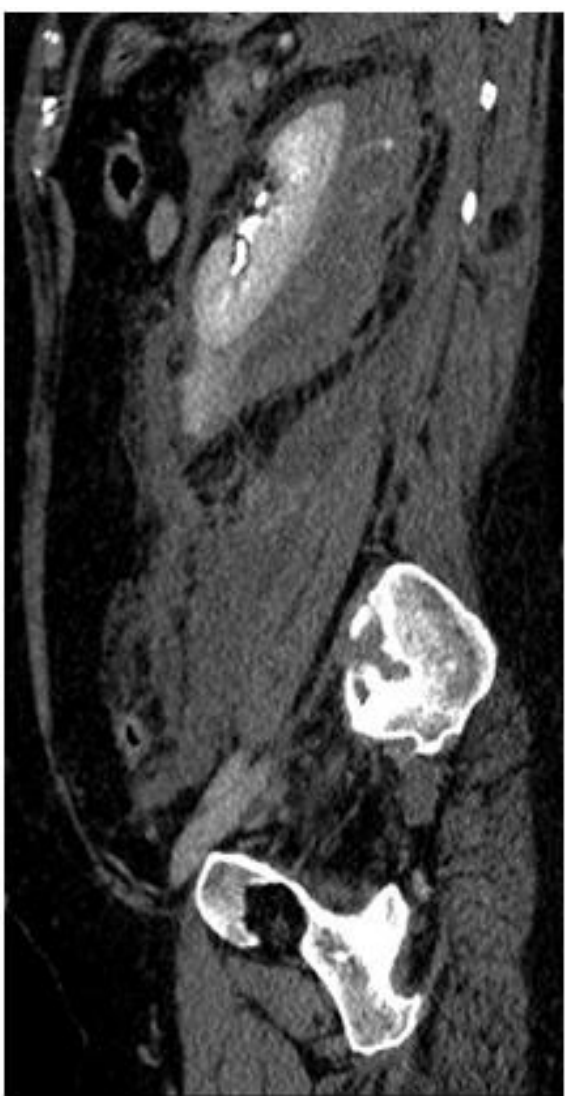

Fig: 1. Axial CECT in arterial (a) and venous (b) phase showing left subcapsular and perinephric heterogeneous collection with arterial extravasation of IV contrast which shows progressive pooling in venous phase (b). Coronal reformatted images in arterial(c), venous (d) and delayed (e) images showing active contrast extravasation and progressive filling and heterogeneity in further images. Note also to be made of the extent of the collection inferiorly extending upto the pelvic brim.

\section{Intramuscular Hematoma}

The patient is a 90 year old priest who had presented with pain and increase in girth of left forearm. He had a recent episode of unstable angina for which he was put on heparin. An MRI evaluation was done. The study reveals an ill defined hyperintensity in the subcutaneous fat of left arm. There is diffuse increase in the bulk of the muscles of the posterior compartment of arm i.e. triceps with ill defined heterogeneous inter and intramuscular collection which shows predominant $\mathrm{T} 1$ hyper intensity and $\mathrm{T} 2$ hyperintensity and heterogeneous post contrast enhancement. The collection shows areas of blooming on MERGE sequence. All these features suggested the possibility of a hematoma.
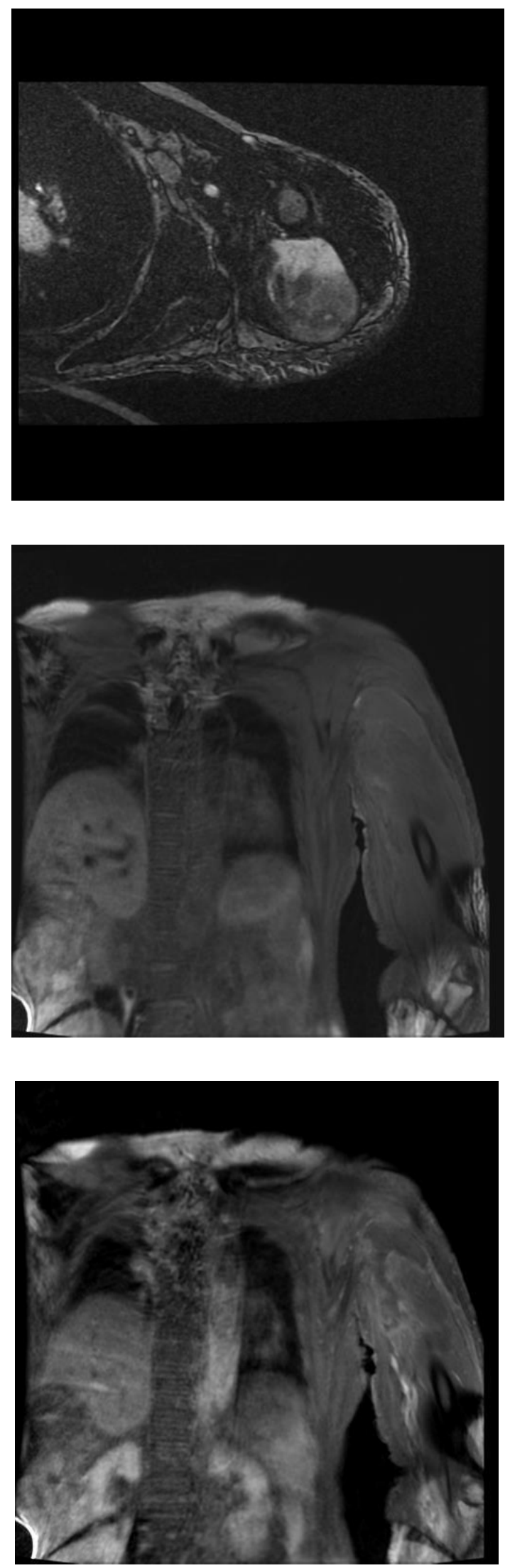


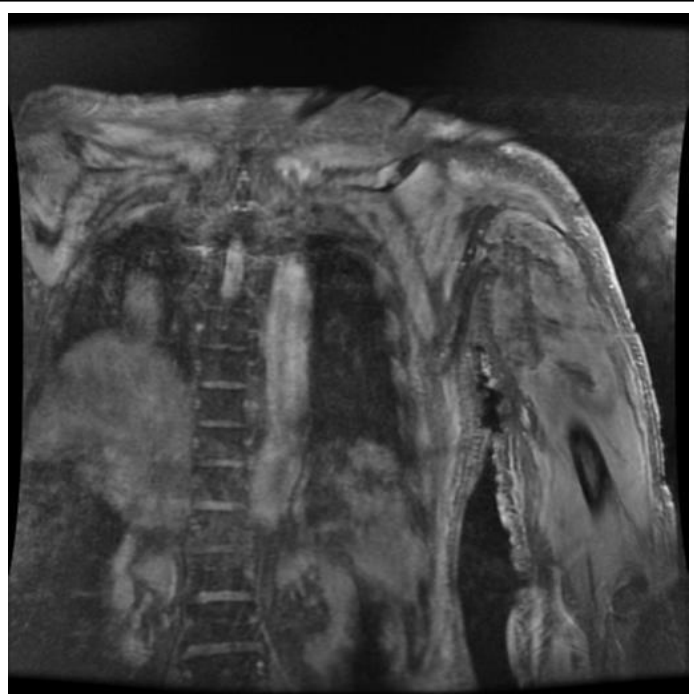

Fig: 2. Axial T2 weighted (a), Pre contrast (b) and post contrast (c) coronal $\mathrm{T} 1$ sequences and Coronal MERGE (d) sequences showing T1 slightly hyper and $\mathrm{T} 2$ hyperintense area involving the posterior compartment of left arm involving the triceps muscle showing heterogeneous enhancement and blooming in MERGE sequences.

\section{Intramural Oesophageal Hematoma}

This 76 year old male had presented with chest pain and dysphagia. He was put on Warfarin for chronic DVT. A CT chest examination was done which revealed, well defined hypodense intramural filling defect with intraluminal component in the mid and distal thoracic esophagus with hyperdense fluid level. The lesion is seen to arise from the posterior and right lateral wall, extending into the cardia of stomach. The lesion shows no significant enhancement on postcontrast study with eccentric crescent of air and contrast in the anterior and left lateral aspect. There is focal air in the mediastinum on right side. There is dilatation of esophagus proximal to the lesion with contrast stasis suggestive of partial obstruction. The patient also had bilateral pleural effusion and minimal mediastinal air. All these gave us the possibility of a dissecting intramural hematoma of oesophagus. The presence of pleural effusion and the mediastinal air should raise the suspicious of possible rupture.
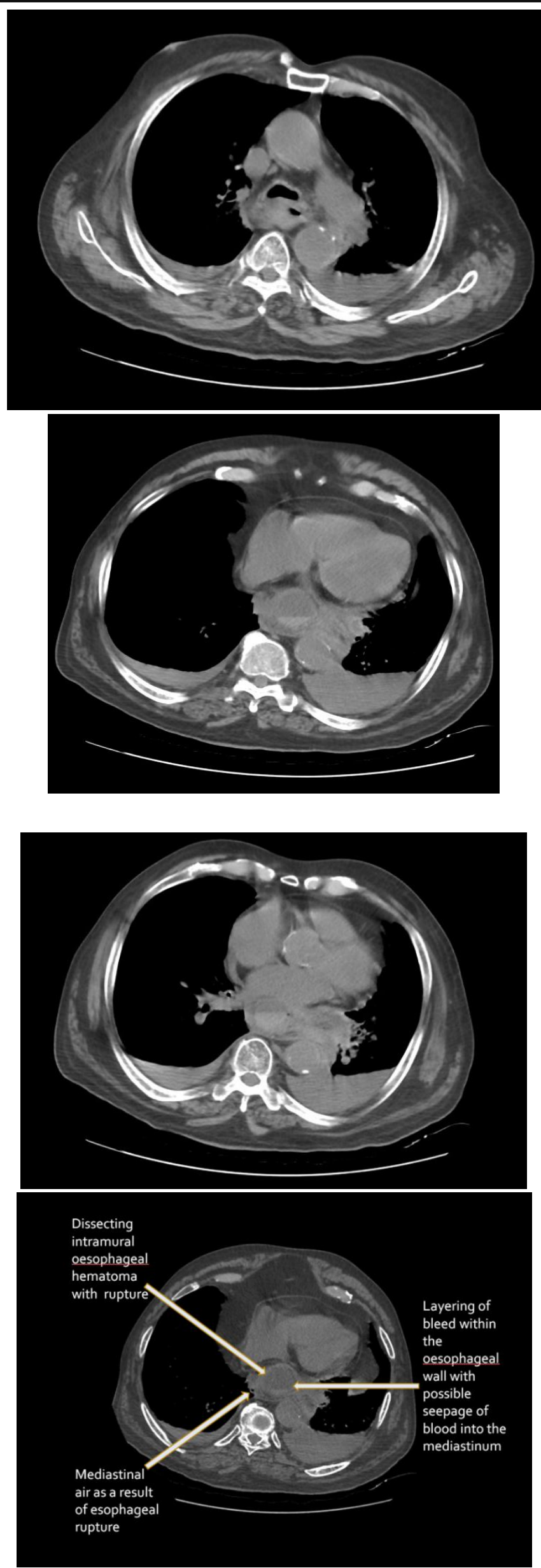


\section{JMSCR Vol||09||Issue||11||Page 187-195||November}

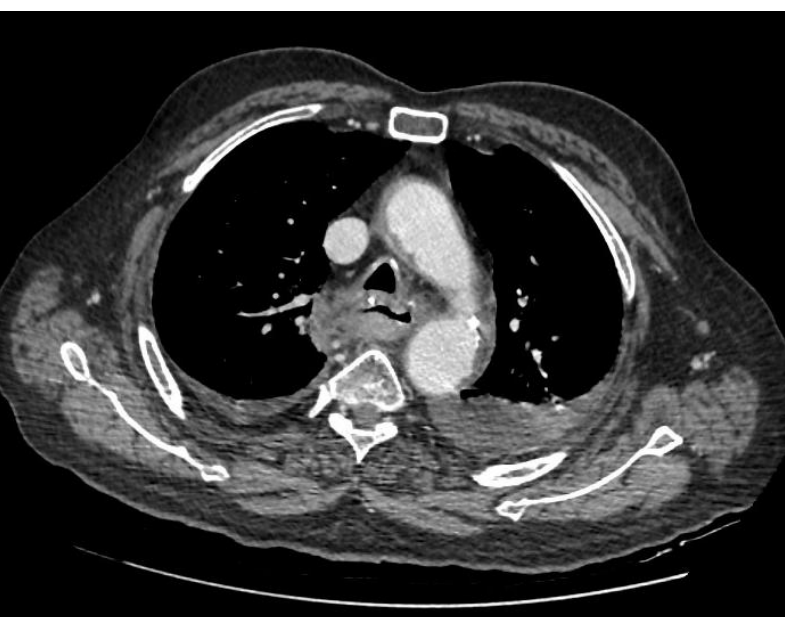

Fig: 3. Plain (a-d) and post contrast axial CT sections at multiple levels of thorax showing a well defined hypodense filling defect in mid and lower thoracic esophagus with hyperdense dependant fluid level with no significant post contrast enhancement. Possibility of dissecting intramural esophageal hematoma was raised with possible rupture owing to the presence of mediastinal free air and bilateral mild pleural effusion.

\section{Abdominal Wall Hematoma}

This is a 47 year old female who had presented with pain involving right suprapubic region. She was put on LMWH for a recent episode of TIA. An MRI evaluation of the abdomen and pelvis was done which revealed, a heterogeneously hyperintense $\mathrm{T} 2 \mathrm{~W}$, iso to mildly hyperintense T1W signal intensity area showing mild blooming on SWAN sequence involving the distal aspect of right rectus abdominis muscle measuring about 3 $\mathrm{x} 2.6 \mathrm{~cm}$. Adjacent muscle oedema is seen. There is also muscle oedema of the left rectus abdomen is muscle in the distal aspect. The rectus sheath is however intact. These findings are consistent with rectus muscle hematoma.
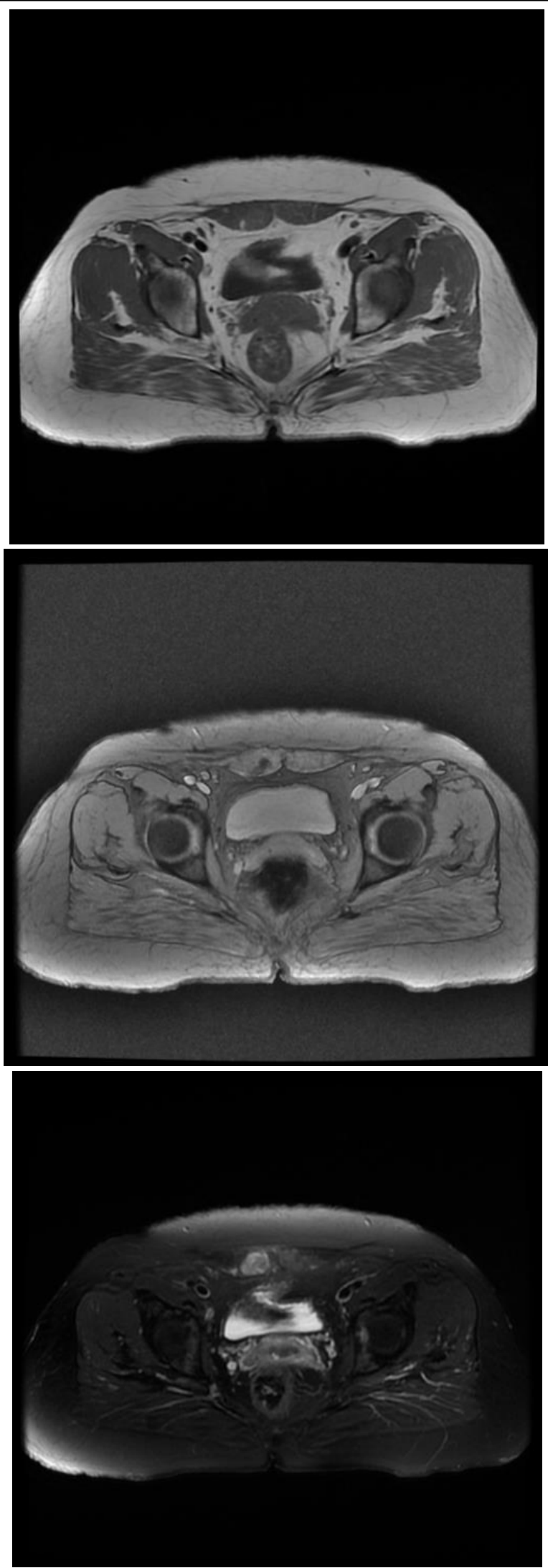

Fig: 4. Axial T1 (a), MERGE (b) and T2 FS (c) sequences showing a mildly $\mathrm{T} 1$ hypertintense, heterogeneously $\mathrm{T} 2$ hyperintense area noted in right rectus muscle in suprapubic location showing blooming in MERGE sequences with mild adjacent perilesional muscular edema. 


\section{JMSCR Vol||09||Issue||11||Page 187-195||November}

\section{Aortic Intramural Hematoma}

This 61 year old male patient came with c/o chest pain radiating to the back with bilateral lower limb claudication. He was on long term warfarin for atrial fibrillation. An aortic angiogram done for him reveals a crescentic high attenuating area of eccentric thickening of aortic wall (high attenuation crescent sign) which is seen extending from ascending aorta till bilateral common iliac arteries which represents an intramural hematoma of aortic wall. These region is showing absent contrast enhancement on post contrast images. There is no obvious evidence of intimal flap as well. All the major aortic branches are seen to be arising from the contrast opacified portion of the lumen. So the possibility of an aortic dissection with thrombosed lumen is less likely.
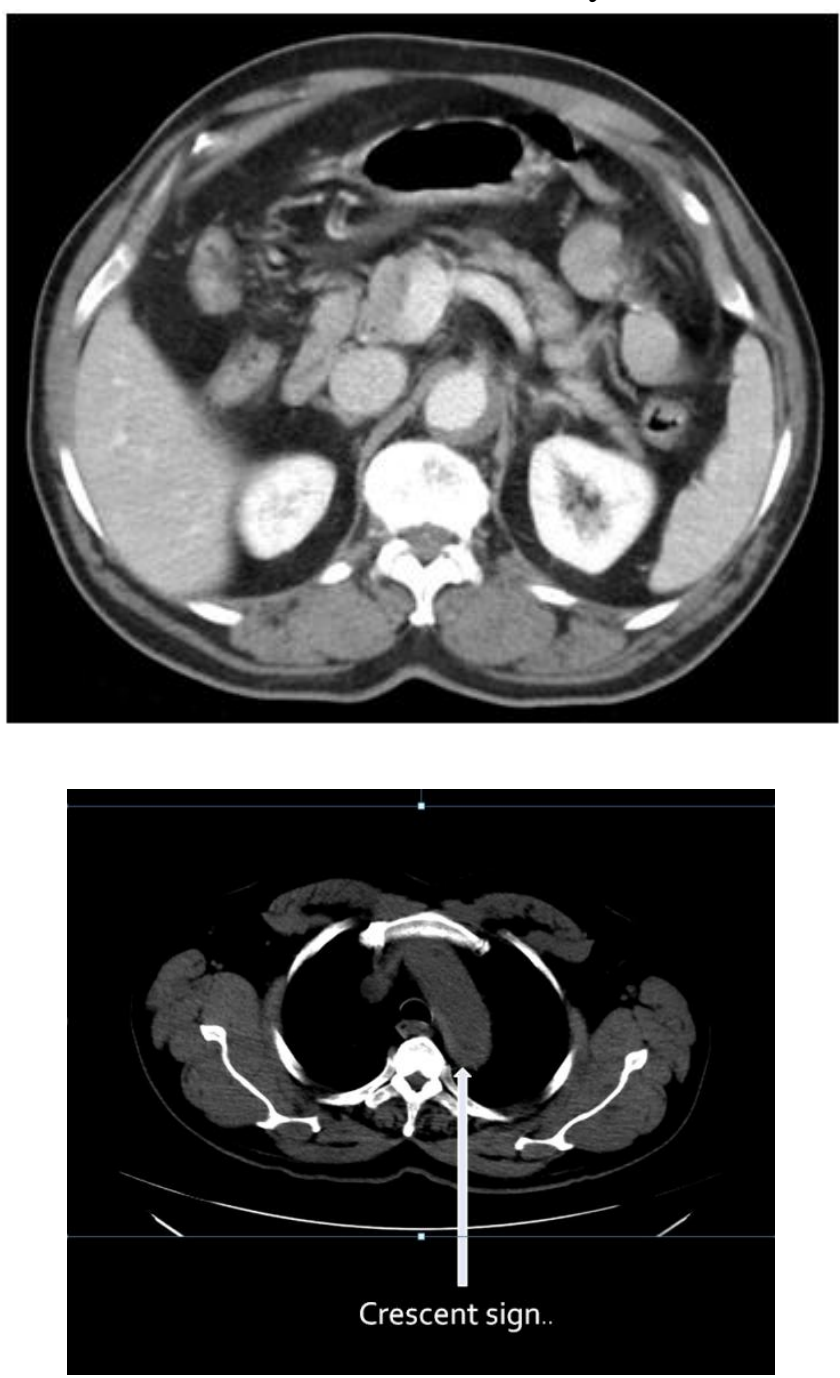
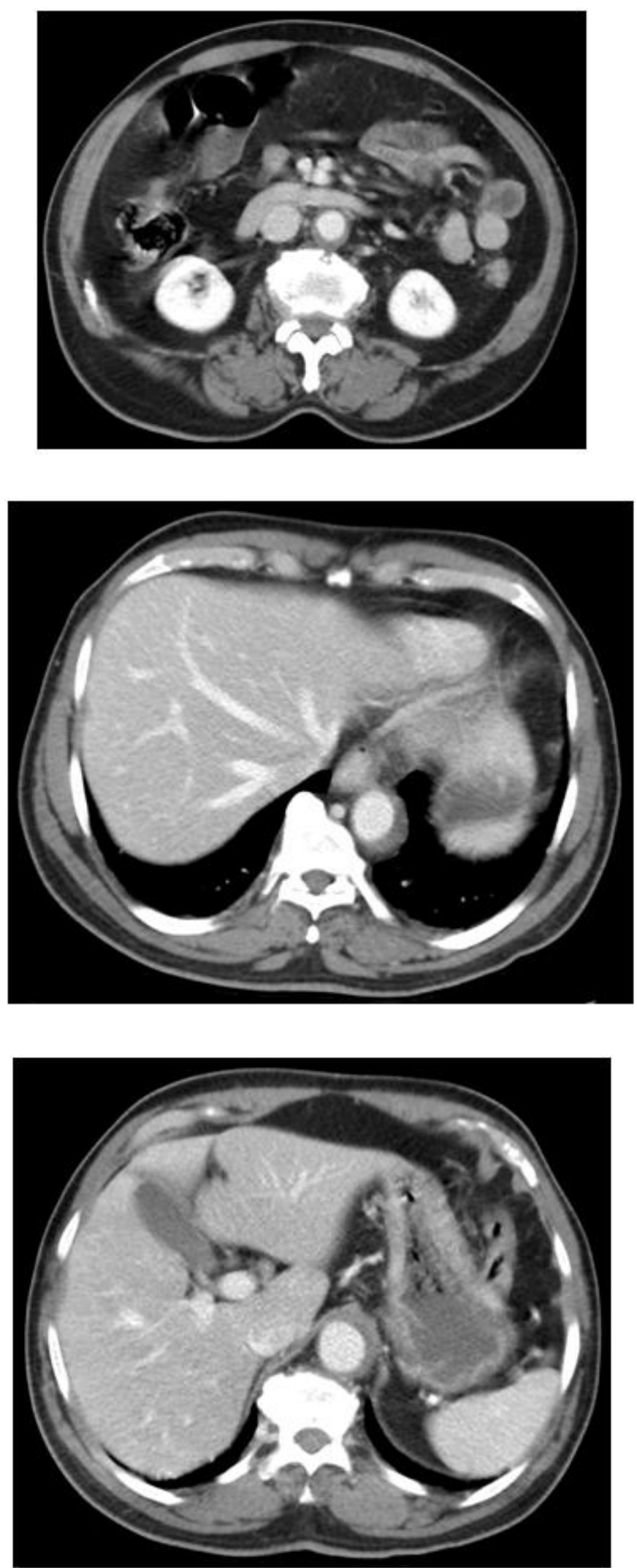


\section{JMSCR Vol||09||Issue||11||Page 187-195||November}

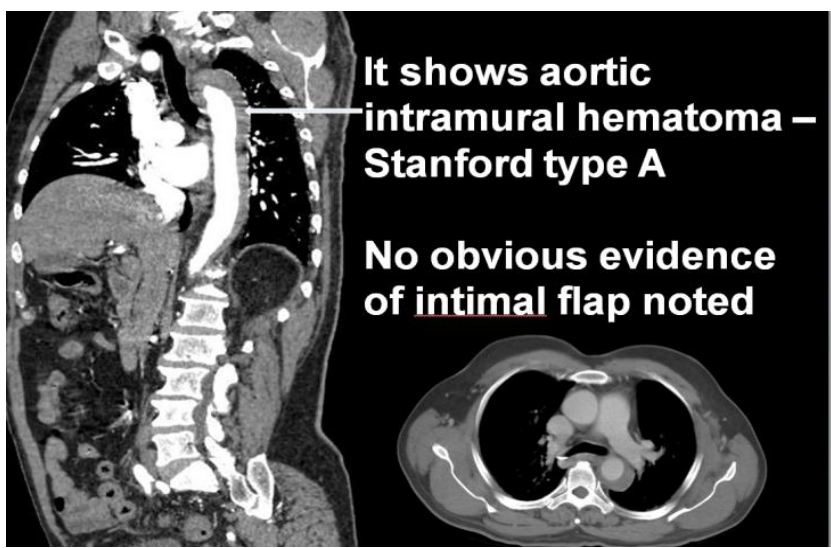

Fig: 5. Plain axial (a) and CECT Axial images (b$\mathrm{d}, \mathrm{f})$ Coronal reformatted images (e) showing a crescentic high attenuation area of eccentric thickening of aortic wall in its posterolateral wall predominantly, which does not show any post contrast enhancement.

\section{Bilateral Perinephric Hematoma}

This 49 year old male patient has been undergoing repeated hemodialysis for his chronic renal compromise which was not heparin free. Now he has presented with abdominal pain and hematuria. CECT abdomen has been performed which revealed extensive ill defined irregularly outlined areas with marked heterogenity having soft tissue and intermixed fatty attenuation, in bilateral perinephric regions $(\mathrm{R}>\mathrm{L})$ with minimal extension to bilateral anterior pararenal spaces and pelvis. There is no obvious extension to posterior pararenal regions. This is seen to displace the uncinate process of pancreas, duodenum, ascending and descending colons anteriorly and mildly towards the midline. Both kidneys appeared shrunken.

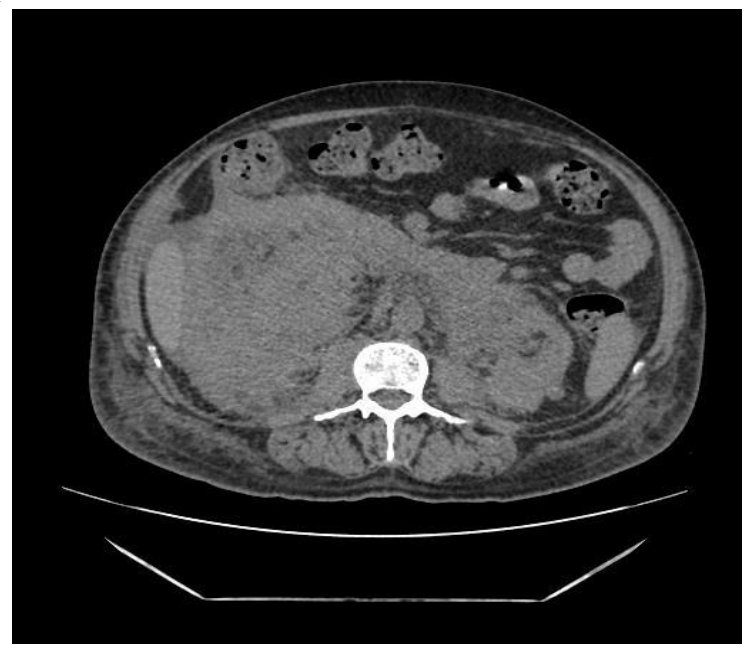

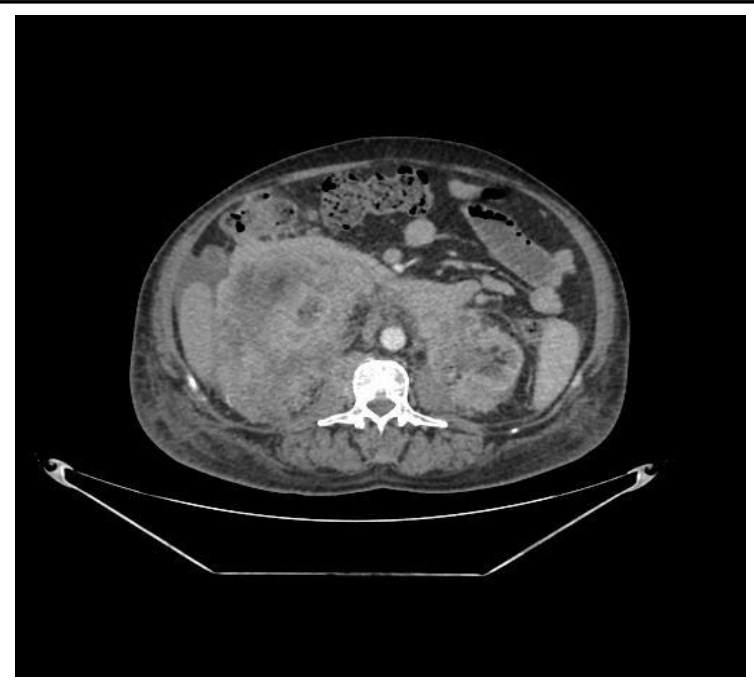

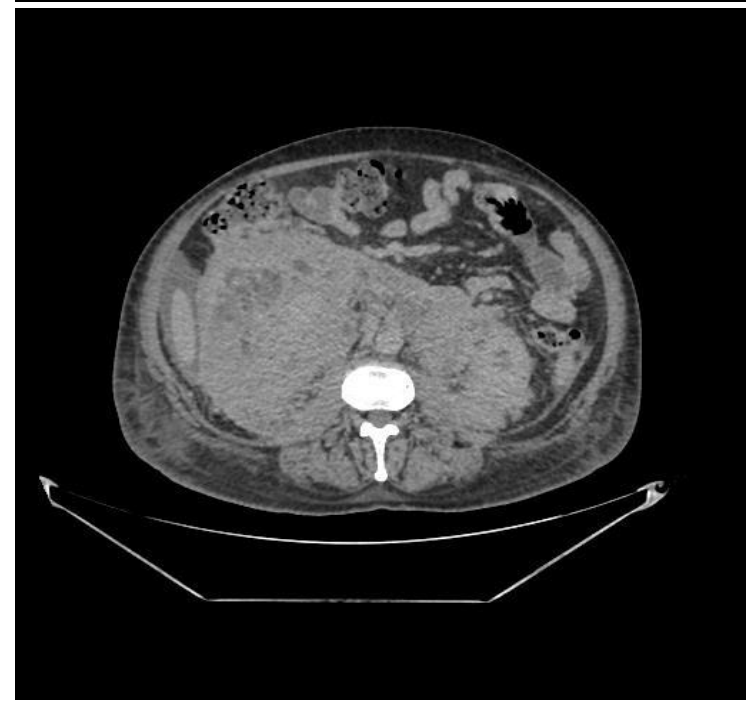

Fig: 6. Axial Plain (a), post contrast arterial (b) and delayed (c) CT images of abdomen at the kidney level showing heterogeneous, irregularly outlined, ill defined soft tissue with intermixed fat density in bilateral perinephric region with extension into para renal space with subtle contrast enhancement of renal parenchyma and no contrast excretion in both kidneys. Suggestive of perinephric hematoma.

\section{Conclusion}

Medical practitioners may resort to anticoagulant administration in various clinical settings and can lead to multitude of hemorrhagic manifestations as mentioned above. The anticoagulant dose should be properly titrated with close monitoring of PT/INR values and as medical professionals and imageologists, we radiologists should always expect such hemorrhagic incidences in patients taking anticoagulants. 


\section{References}

1. Linkins LA, Choi PT, Douketis JD. Clinical impact of bleeding in patients taking oral anticoagulant therapy for venous thromboembolism: A metaanalysis. Ann Intern Med 2003;139:893900.

2. Hylek EM. Complications of oral anticoagulant therapy: Bleeding and nonbleeding, rates and risk factors. Semin VascMed 2003;3:271-8.

3. Beyth RJ. Hemorrhagic complications of oral anticoagulanttherapy. ClinGeriatr Med 2001;17:49-56.

4. Jacobs LG. Warfarin pharmacology, clinical management, and evaluation of hemorrhagic risk for the elderly. Cardiol Clin2008;26:157-67, v.

5. Nazarian LN, Lev-Toaff AS, Spettell CM, et al. CT assessment of abdominal hemorrhage in coagulopathic patients: Impact on clinical management. Abdom Imaging 1999;24: 246-9.

6. Furlan A, Fakhran S, Federle MP. Spontaneous abdominal hemorrhage: Causes, CT findings, and clinical implications. Am J Roentgenol 2009;193:1077-87.

7. Schulman S, Beyth RJ, Kearon C, et al. Hemorrhagic complications of anticoagulant and thrombolytic treatment: American College of Chest Physicians Evidence-Based Clinical Practice Guidelines (8th Edition). Chest 2008;133(6 suppl): 257S-98S.

8. Pretorius ES, Fishman EK, Zinreich SJ. CT of hemorrhagic complications of anticoagulant therapy. J Comput Assist Tomogr 1997;21:44-51.

9. Seseke S, Schreiber M, Rebmann U, Seseke F. Spontaneperirenale Hämatomebei Patientenmit Koagulopathenoderunter Antikoagulanzientherapie [Spontaneous perirenal hematomas in patients taking anticoagulation medication or having a bleeding diathesis]. Aktuelle Urol. 2008 May;39(3):215-8. German. doi: 10.1055/s-2007-1016433.

PMID: 18478495 .

10. Cañadas MV, Vilacosta I, Ferreirós J, Bustos A, Díaz-Mediavilla J, Rodríguez E. Intramural aortic hematoma and anticoagulation. Revista Española de Cardiología (English Edition). 2007 Jan 1;60(2):201-4.

11. Subhash R, Unnikrishnan G, Balakrishnan D, Sudheer OV, Dhar P, Sudhindran S. Gastrointestinal intramural hematoma-analysis of clinical and radiological features for early differentiation from mesenteric ischemia. Indian J Gastroenterol. 2014 Jul;33(4):364-8. doi: 10.1007/s12664-014-0449-z. Epub 2014 Mar 28. PMID: 24671723. 